\title{
Echocardiography in COVID-19 Pandemic Clinical Findings and the Importance of Emerging Technology
}

\author{
Alberto Barosi, MD ${ }^{a, *}$, Luca Bergamaschi, MD ${ }^{a}$, Ignazio Cusmano, MDa, \\ Alessio Gasperetti, MD ${ }^{a, b}$, Marco Schiavone, MDa , Elisa Gherbesi, MD ${ }^{a}$
}

\section{KEYWORDS}

- COVID-19 • Echocardiography • Myocardial strain • Ultramobile devices

\section{ECHOCARDIOGRAPHY IN COVID-19 PATIENTS}

Coronavirus disease 2019 (COVID-19) mainly affects the respiratory system, ${ }^{1}$ but important amount of evidence has described cardiac involvement, ${ }^{2,3}$ in up to $20 \%$ of patients, ${ }^{4}$ besides collaterally leading toward a change in hospital admission patterns for other cardiac diseases. ${ }^{5-7}$ Echocardiography, as a widely available, costeffective tool for the evaluation of cardiac structure and function, can provide important information that can affect the management of COVID-19 patients, but considering the risk of equipment contamination and personnel exposure, ${ }^{8}$ focused evaluations instead of complete echocardiograms and the use of portable devices easy to disinfect, are recommended.

The present review discusses the principal echocardiographic findings in COVID-19 patients (Fig. 1; Table 1), practical aspects, and the role of emerging technology.

\section{LEFT AND RIGHT VENTRICLE: MAJOR FINDINGS}

COVID-19 can cause a wide range of cardiac conditions, which include acute myocardial infarction, ${ }^{9-11}$ takotsubo cardiomyopathy, ${ }^{12}$ myocarditis, ${ }^{13}$ arrhythmogenic, ${ }^{14}$ thrombotic manifestations, ${ }^{15}$ and potential drug-related effects, ${ }^{16-18}$ as documented by several studies and early case reports.
However, the incidence of the cardiac involvement and the subsequent implications for treatment and resource allocation for the screening of these conditions are not well defined, but data confirmed that cardiac injury is associated with increased mortality in COVID-19. ${ }^{19,20}$

Regarding myocardial infarction, Stefanini and colleagues collected, in a national registry, 28 COVID-19 patients with ST-elevation myocardial infarction (STEMI): in approximately $40 \%$ of them, a culprit lesion was not detected by coronary angiography, pointing out the role of cytokine storm, hypoxic injury, coronary spasm, microthrombi, direct endothelial, or vascular injury. On echocardiography, about $80 \%$ of patients had localized left ventricular (LV) wall motion abnormalities, whereas left ventricular ejection fraction (LVEF) was less than $50 \%$ in about $60 \%$ of patients. $^{21}$

In COVID-19 have also been described cases of typical and atypical Takotsubo syndrome, ${ }^{22}$ possibly triggered by emotional stress and physical stress by infection itself, with a significant increase in the incidence of stress cardiomyopathy when compared with prepandemic periods ${ }^{23}$ : echocardiography has a diagnostic role in detecting the typical apical ballooning and possible unfavorable findings (LV outflow tract obstruction, mitral regurgitation, apical thrombus). ${ }^{24}$

Of particular interest, is COVID-19-related myocarditis: a direct cardiotropic localization of

\footnotetext{
${ }^{a}$ Cardiology Unit, Luigi Sacco University Hospital, Milan, Italy; ${ }^{\text {b }}$ Division of Cardiology, Johns Hopkins University, Baltimore, MD, USA

* Corresponding author. Department of Cardiology, University Hospital Luigi Sacco, Milan, Italy.

E-mail address: barosi.alberto@asst-fbf-sacco.it
} 


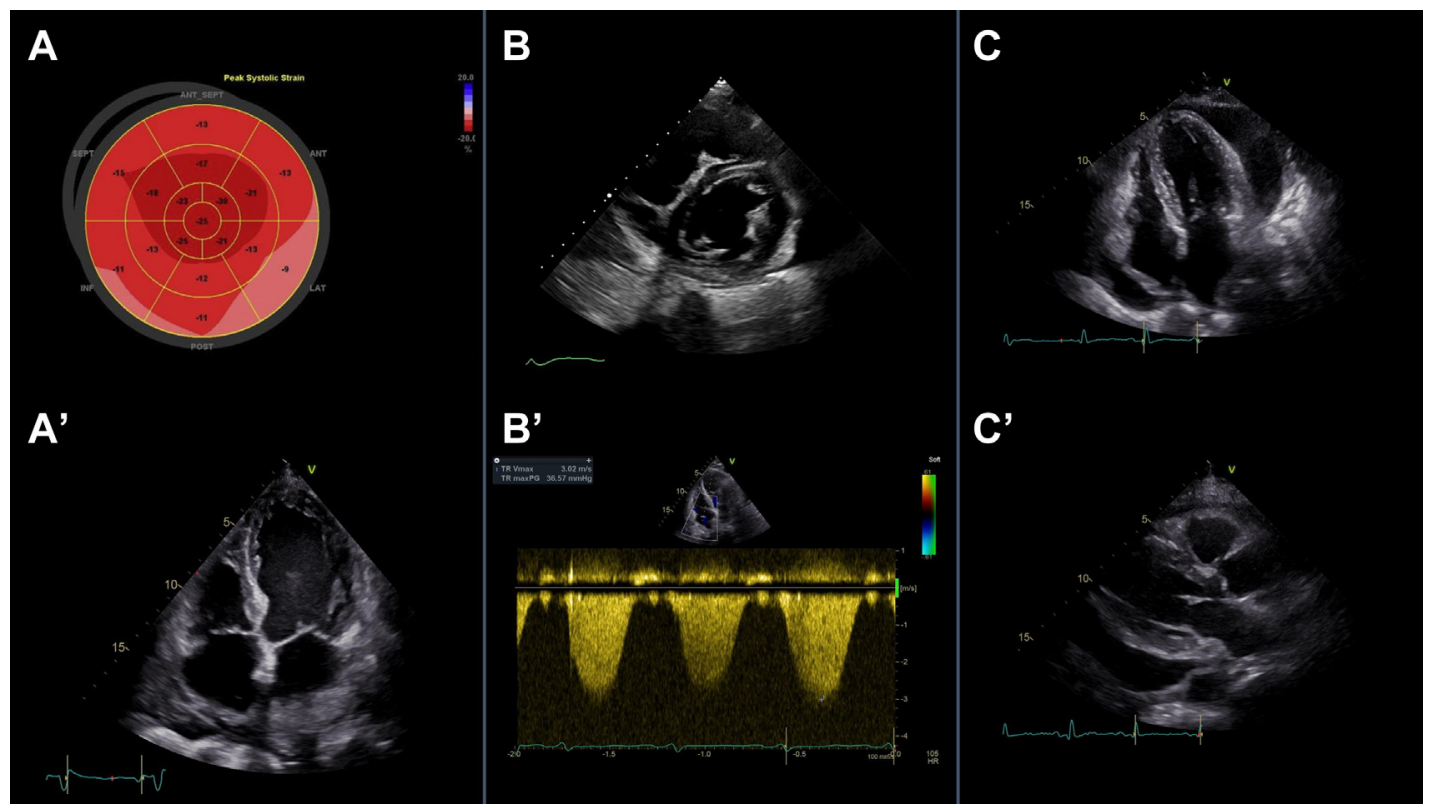

Fig. 1. Example of echocardiographic findings in different clinical settings of myocardial involvement in COVID19 patients. Panel $A$ and $A^{\prime}$ : Reduced regional myocardial strain in lateral wall and dilation of left ventricle in myocarditis; Panel B and B': D-shape of left ventricle and increase in tricuspid regurgitation jet velocity in pulmonary embolism; Panel $C$ and $C^{\prime}$ : pericardial effusion, in parasternal long-axis and four-chamber view.

SARS-CoV-2 into myocytes has never been described, but some autoptic findings (lymphocyte infiltrates and macrophagic response) resulted compatible with viral myocarditis. ${ }^{25}$ In a systematic review of Sawalha and colleagues

\begin{tabular}{|c|c|}
\hline \multicolumn{2}{|c|}{$\begin{array}{l}\text { Table } 1 \\
\text { Characteristics echocardiographic findings in } \\
\text { different clinical settings of myocardial } \\
\text { involvement in COVID-19 patients }\end{array}$} \\
\hline Myocarditis & $\begin{array}{l}\text { - LV dilation } \\
\text { - LV pseudohypertrophy } \\
\text { - LV diffuse hypokinesis } \\
\text { - Reduction in LVEF }\end{array}$ \\
\hline $\begin{array}{l}\text { Acute coronary } \\
\text { syndromes }\end{array}$ & $\begin{array}{l}\text { - Regional LV wall-motion } \\
\text { abnormalities } \\
\text { - Mechanical complications }\end{array}$ \\
\hline $\begin{array}{l}\text { Pulmonary } \\
\text { embolism }\end{array}$ & $\begin{array}{l}\text { - RV dilation with D-shape of } \\
\text { LV } \\
\text { - RV dysfunction } \\
\text { - McConnell's sign } \\
\text { - Increase in sPAP } \\
\end{array}$ \\
\hline Pericarditis & $\begin{array}{l}\text { - Pericardial effusion, } \\
\text { possible cardiac tamponade } \\
\text { - Brightness of pericardium }\end{array}$ \\
\hline
\end{tabular}

Abbreviations: LV, left ventricle; LVEF, left ventricular ejection fraction; RV, right ventricle; SPAP, systolic pulmonary arterial pressure. comprising 14 cases with myocarditis/myopericarditis believed to have occurred secondary to COVID-19 infection, echocardiography was performed in most cases (83\%) and 60\% had reduced LVEF, with diffuse hypokinesis in $30 \%$ of patients. $^{26}$

However, excluding specific clinical scenario, while big alterations at conventional echocardiography could be difficult to detect, ${ }^{27}$ deformation imaging can be the appropriate tool to identify subclinical modifications $^{28}$ : in a study of Stobe and colleagues, despite normal LVEF, most of the infected patients (that ranged from mild to severe symptoms) showed abnormal LV deformation, in particular a reduced longitudinal strain observed predominantly in more than one basal LV segment (in 10/14 patients, 71\%), that was attributed to a possible subepimyocardial involvement of SARS-CoV-2-induced myocarditis, confirmed by cardiac magnetic resonance (CMR) but only in 2 patients. ${ }^{29}$ Left ventricular-global longitudinal strain (LV-GLS) was altered in up to $80 \%$ of patients hospitalized for COVID-19 infection ${ }^{30}$ (32/40 patients, mean LV-GLS of $12.1 \% \pm 4.0$, normal $<16 \%$ ) and was superior to LVEF for predicting adverse outcome. ${ }^{31}$ Indeed, an important role of echocardiography could be the prognostic stratification of patients: LV-GLS was found to be an independent predictor of mortality through multivariate analysis. ${ }^{32-34}$ 
A major role in COVID-19 is played by the right ventricle (RV): it could be affected secondarily to elevation in RV afterload; increases in PVR causes RV dilation and eventual RV failure, which has been related to a worse prognosis. ${ }^{35}$

COVID-19 could cause acute respiratory distress syndrome (ARDS) through vasoactive mediators, vascular thrombosis, and vascular compression secondary to atelectasis and edema. ${ }^{36}$

Moreover, patients with COVID-19 are in a prothrombotic state that predisposes them to thromboembolic events, including deep vein thrombosis and pulmonary embolism (PE). ${ }^{37-39}$

The study by Dweck and colleagues, a survey of the European Association of Cardiovascular Imaging, found that $33 \%$ of patients had an abnormal RV on echocardiography. ${ }^{40}$ Of these subjects, $19 \%$ had mild-to-moderate RV dysfunction and $6 \%$ had severe dysfunction (of note, the index used for the definition of dysfunction was not specified). The RV was dilated in $15 \%$, a D-shaped LV was seen in $4 \%$ and pulmonary artery pressure was elevated in $8 \%$.

In the study by Mahmoud-Elsayed and colleagues, $41 \%$ of patients had a dilated RV (RV basal diameter $>41 \mathrm{~mm}$ ) and $27 \%$ had a decreased RV function (fractional area change $<35 \%$ or a tricuspid annular plane systolic excursion $<17 \mathrm{~mm}$ ); most patients had severe respiratory failure and $82 \%$ were on invasive mechanical ventilation. A PE was detected in $20 \%$ of subjects with RV dysfunction as compared with $2 \%$ in those without RV dysfunction. ${ }^{41}$

Jain and colleagues found that $15.3 \%$ of the patients had an increased RV size (12.5\% were mildly increased and $2.8 \%$ were moderately increased). ${ }^{42} \mathrm{RV}$ systolic function (assessed semiquantitatively) was mildly decreased in $26.4 \%$, moderately decreased in $9.7 \%$, and severely decreased in $4.2 \%$.

Several studies reported an association between right-side TTE parameters and prognosis in patients with COVID-19.

Kim and colleagues in an analysis from a US multicentre retrospective study reported that adverse RV remodeling (dysfunction/dilation) conferred a $>2$ fold increase in mortality risk. ${ }^{43}$

Szekely and colleagues ${ }^{44}$ found that the most frequent abnormality among patients with clinical deterioration during follow-up was RV dilatation (with or without dysfunction). In univariable analysis, they reported that shorter pulmonary acceleration time $(<100 \mathrm{msec})$ was associated with clinical deterioration and that RV end-diastolic area was associated with mortality. ${ }^{44}$
$\mathrm{Li}$ and colleagues reported that RV global longitudinal strain was a powerful predictor of death in patients with COVID-19. ${ }^{45}$

In a recent systematic review, Messina and colleagues analyzed studies available in literature and concluded that they have highly variable sample sizes and reported highly heterogeneous findings: LVEF does not seem significantly affected (reported as higher than $50 \%$ in most subjects), LV diastolic function has not been properly assessed and RV dysfunction seems frequent but defined with variable criteria, making it difficult to establish a clear association with higher mortality. ${ }^{46}$

\section{CARDIAC VALVES AND PERICARDIUM}

The presence of valvular heart disease (VHD) has been described in COVID-19 patients undergoing echocardiography during the hospital stay. ${ }^{40}$ These valvular diseases more likely were present before the onset of SARS-CoV-2 infection. A recent study regarding echocardiographic findings among COVID patients reported that half of them presented a significant tricuspid regurgitation followed by aortic regurgitation and mitral regurgitation. ${ }^{47}$ Tricuspid regurgitation is the most common VHD reported in COVID-19 patients and its severity could directly reflect the impairment of the pulmonary circulation during the infection resulting in pulmonary hypertension, especially in patients with severe pneumonia and respiratory insufficiency. ${ }^{48}$

A higher concern, especially in COVID-19 patients in the intensive care unit, is the presence of infectious endocarditis (IE). Some clinical reports have reported endocarditis in a minority prevalence and the presence of valve vegetations were also described in autopsy findings for SARSCoV-2 patients. ${ }^{40,49}$ In this setting, endocarditis is related to bacterial endocarditis or the presence of thrombus; less likely could be caused by the direct SARS-CoV-2 infection and there is no evidence about that relationship.

According to the American Society of Echocardiography (ASE) and the European Association of Cardiovascular Imaging (EACVI) recommendations, in case of suspected or confirmed SARSCoV-2 infections, cardiac imaging should be considered only on an individual basis when it could change the clinical management or be lifesaving for the patients, because of the higher risk of contamination for health care providers, especially during transesophageal echocardiography (TOE). ${ }^{50,51}$ Despite that, TOE remains the gold standard for the diagnosis of IE even in the current COVID-19 pandemic and to better evaluate the severity of a significant VHD. ${ }^{52,53}$ In conclusion, 
in COVID-19 patients with high suspicion of IE or significant VHD, explorative TTE and if inconclusive a subsequent TOE is mandatory for the diagnosis with all the precautions aimed at avoiding the spread of the infectious disease.

Some case reports reported acute pericarditis during SARS-COV-2 infection. ${ }^{54}$ This type of cardiac involvement is often seen in patients with troponin elevation in the context of myocardial damage and less frequently as a primary presentation of COVID-19 infection, which occurred most in younger patients. ${ }^{55,56}$ Pericardial involvement may be driven by the systemic and local inflammatory response to infection and less likely by direct virus damage. ${ }^{57}$ Another form of pericardial involvement is pericardial effusion, found in nearly $5 \%$ of COVID-19 patients who underwent chest CT.

As recommended by ESC guidelines, also in patients with SARS-CoV-2 infection, TTE is recommended as the first diagnostic tool to evaluate pericardial diseases and it is most useful to address the severity of significant effusion. ${ }^{58}$ As second step, in selected cases because of logistic issues in this setting, CMR can evaluate pericardial thickening or small effusions, assess myocardial damage, and precisely define pericardial inflammation. ${ }^{59}$

\section{PRACTICAL ASPECTS}

Since the beginning of the pandemic, concerns have been raised about the risk of performing echocardiograms for the risk of personnel infection, with the subsequent need to select patients in which echocardiography can have a fundamental diagnostic/prognostic role. The principal echocardiography societies released recommendations to review the appropriateness of every examination, favor the use of handheld devices (easier to disinfect) and perform fast and focused evaluations: they provided a modified point-ofcare ultrasound (POCUS) protocol for the evaluation of patients with suspected or confirmed COVID-19, when is likely to have an impact on patient management (summary recommendations in Box 1). ${ }^{50,60}$ Adopting protocols aimed at reducing the number of inappropriate studies, the workflow in echocardiography laboratories has declined by $50 \%$ and the study appropriateness has significantly increased. ${ }^{61}$ Moreover, the use of limited tablet-based echocardiograms can reduce the study time by $79 \% .{ }^{62}$ Considering the importance of prone position in patients developing ARDS, some reports have suggested that prone position echocardiography might be feasible, allowing RV and LV evaluation in a four-chamber view. ${ }^{63}$

\section{Box 1}

Summary recommendations for echocardiography in COVID-19 patients

Indications

- Perform urgent/emergent examinations (that can change patient management or be lifesaving, in particular for TOE)

- Defer elective/routine follow-up examinations

Personnel protection

- Droplet precautions for TTE (protective clothing, gloves, headcovers, specific facemasks, and eye shields)

- Airborne precautions for TOE (N-95 or N-99 respirator masks)

- Patients should wear a surgical mask during imaging

Method

- Focus cardiac ultrasound study:

LV: LVEF, regional dysfunction, enddiastolic cavity dimension

RV: TAPSE, end-diastolic cavity dimension, tricuspid regurgitation pressure gradient

Valves: gross signs of valvular disease

Pericardium: thickening or effusion

- ECG monitoring can be omitted

Technology

- Prefer hand-held or smaller lap-top-based scanners

- Measurements should be performed offline

Abbreviations: LV, left ventricle; LVEF, left ventricular ejection fraction; RV, right ventricle; TAPSE, tricuspid annular plane systolic excursion; TOE, transesophageal echocardiography; TTE, transthoracic echocardiography.

\section{DEVELOPMENT OF NEW TECHNOLOGIES}

The need for absolute isolation of symptomatic patients and the high possibility of spreading the infection outside the isolation rooms in the highintensity care departments, makes any attempt at an instrumental diagnostic approach difficult, given also the absolute need to decontaminate the equipment used after each individual examination. The advantages deriving from the execution of echocardiography examination at the patient's bedside are well known but the high risk of serious coronavirus infection diffusion has made its use difficult. Disinfection is the only way to counter the risk of spreading the disease from one patient to another 
or among the operators themselves, which event is even more dangerous because it would jeopardize the tightness of the system. Hand-carried echocardiography devices offer rapid and readily available information at the bedside, as they help overcome the problems caused by the use of cumbersome standard equipment. 62,64 The use of "ultramobile devices" has recently been introduced in the area of cardiovascular ultrasound diagnostics. These devices are miniaturized systems equipped with diagnostic quality two-dimensional (2D) and color Doppler imaging. With simplified criteria, they can detect LV dysfunction and moderate-severe valvulopathy with good sensitivity and specificity. They are extremely useful in the immediate diagnosis of pericardial effusion and in the definition of states of cardiocirculatory failure or inflammatory states of pulmonary parenchyma. In resource-limited settings, ultramobile systems can reduce the need for standard echocardiography. "Ultramobile" systems have a probe that can be connected to any handheld device, smartphone, or tablet, allowing the execution of cardiac, pulmonary, vascular, abdominal, soft tissue, and joint ultrasound. The isolation system, required to avoid the danger of spreading the infection, is simple, consisting of a mono-use coating sheath commonly used for epicardial echocardiography in the cardiac surgery operating room. At the end of the examination, the probe is removed from the casing, reinserted in a new protective sheath, and ready for a subsequent examination. The compactness of the system makes it easily isolable from the environment with a monouse coating sheath, making it very practical in environments with a high level of contamination or where absolute sterility is required. Thanks to Internet connectivity, the images, stored in DICOM format, can also be simultaneously viewed in real time by other doctors outside the contaminated area: data collection and their quick transfer allow a postprocedural analysis in the safe zone. Finally, a video call system allows communication between operators. Moreover, this aspect gives the possibility to exploit few medical imaging experts for the interpretation of examinations from numerous Spot Centers, also performed by doctors or trained nonmedical staff not specialized in imaging.

Moreover, in China, a pilot study of robotassisted teleultrasound based on 5G Network was conducted $^{65}$ : ultrasound specialists carried out the robot-assisted teleultrasound, manipulating a handheld controller, which can control the robotic arm, and did remote consultation in order to settle the problem of early cardiopulmonary evaluation in COVID-19 patients.

A point of particular importance is the role of artificial intelligence $(\mathrm{Al})$, technology that creates a computerized model to solve different problems without the requirement of human assistance, continuously learns from the data set, and predicts outcomes accurately. ${ }^{66,67}$ It was used in intensive care unit by critical care physicians without formal training in ultrasound to obtain POCUS images, with the use of real-time prescriptive guidance to direct the physician's transducer position and hand movements to acquire the images, automatically capturing them when appropriate, detecting LVEF with high accuracy, and uploading to the archive and communication system for offline review. ${ }^{68} \mathrm{Al}$ includes machine learning (ML), which offers the potential to improve the accuracy and reliability of echocardiography by combining clinician interpretation with information derived from $\mathrm{ML}$ algorithms. In conclusion, high volume data generated from cardiac imaging can be integrated into a multiparametric approach for pattern recognition and imaging data-based disease phenotype characterization, particularly useful and timesaving in the setting on COVID-19 pandemic. ${ }^{69}$

\section{SUMMARY}

Echocardiography has a diagnostic and prognostic role in COVID-19, helping in recognizing cardiac involvement. Considering the high risk of personnel infection and equipment contamination, focused echocardiographic protocols with portable devices are recommended.

Available echocardiographic data on COVID-19 patients actually do not provide definite evidence, due to multiple factors: high heterogeneity between studies in numbers of patients, miscellaneous results, and low quality of echocardiographic studies for technical difficulties in performing bedside procedures (patients with respiratory distress/invasive ventilation; while wearing personal protective equipment).

In conclusion, echocardiography represents an important tool in the management of COVID-19 patients, but more studies with standardized acquisitions and methods are needed to describe definite echocardiographic findings in this setting.

\section{CLINICS CARE POINTS}

- Focused echocardiographic protocols can detect cardiac involvement in COVID-19

- Echocardiography is strictly recommended if it could change the clinical management 


\section{- Available data do not provide definite evi- dence on echocardiographic findings in COVID-19}

\section{REFERENCES}

1. Busana M, Gasperetti A, Giosa L, et al. Prevalence and outcome of silent hypoxemia in COVID-19. Minerva Anestesiol 2021;87(3):325-33.

2. Zheng YY, Ma YT, Zhang JY, et al. COVID-19 and the cardiovascular system. Nat Rev Cardiol 2020;17(5): 259-60.

3. Kang Y, Chen T, Mui D, et al. Cardiovascular manifestations and treatment considerations in COVID19. Heart 2020;106(15):1132-41.

4. Shi S, Qin M, Shen B, et al. Association of cardiac injury with mortality in hospitalized patients with COVID-19 in Wuhan, China. JAMA Cardiol 2020; 5(7):802-10.

5. De Filippo O, D'Ascenzo F, Angelini F, et al. Reduced rate of hospital admissions for ACS during covid-19 outbreak in Northern Italy. N Engl J Med 2020;383(1):88-9.

6. Severino P, D'Amato A, Saglietto A, et al. Reduction in heart failure hospitalization rate during coronavirus disease 19 pandemic outbreak. ESC Hear Fail 2020;7(6):4182-8.

7. De Rosa S, Spaccarotella C, Basso C, et al. Reduction of hospitalizations for myocardial infarction in Italy in the COVID-19 era. Eur Heart J 2020;41(22):2083-8.

8. Schiavone M, Forleo GB, Mitacchione G, et al. Quis custodiet ipsos custodes: are we taking care of healthcare workers in the Italian COVID-19 outbreak? J Hosp Infect 2020;105(3):580-1.

9. Capaccione KM, Leb JS, Belinda D, et al. Acute myocardial infarction secondary to COVID-19 infection: a case report and review of the literature. Clin Imaging 2021;72:178-82.

10. Schiavone M, Gobbi C, Biondi-Zoccai G, et al. Acute coronary syndromes and covid-19: exploring the uncertainties. J Clin Med 2020;9(6):1683.

11. Schiavone M, Gasperetti A, Mancone M, et al. Redefining the prognostic value of high-sensitivity troponin in COVID-19 patients: the importance of concomitant coronary artery disease. J Clin Med 2020;9(10):3263.

12. Gomez JMD, Nair G, Nanavaty P, et al. COVID-19associated takotsubo cardiomyopathy. BMJ Case Rep 2020;13(12):1-5.

13. Zeng JH, Liu YX, Yuan J, et al. First case of COVID19 complicated with fulminant myocarditis: a case report and insights. Infection 2020;48(5):773-7.

14. Mitacchione G, Schiavone M, Gasperetti A, et al. Ventricular tachycardia storm management in a COVID-19 patient: a case report. Eur Heart J Case Rep 2020;4(FI1):1-6.
15. Schiavone M, Gasperetti A, Mancone M, et al. Oral anticoagulation and clinical outcomes in COVID19: an Italian multicenter experience. Int J Cardiol 2021;323:276-80.

16. Gasperetti A, Biffi M, Duru F, et al. Arrhythmic safety of hydroxychloroquine in COVID-19 patients from different clinical settings. Europace 2020;22(12): 1855-63.

17. Chang WT, Toh HS, Liao C Te, et al. Cardiac involvement of COVID-19: a comprehensive review. Am J Med Sci 2021;361(1):14-22.

18. Mitacchione G, Schiavone M, Curnis A, et al. Impact of prior statin use on clinical outcomes in COVID-19 patients: data from tertiary referral hospitals during COVID-19 pandemic in Italy. J Clin Lipidol 2021; 15(1):68-78.

19. Guo T, Fan Y, Chen M, et al. Cardiovascular implications of fatal outcomes of patients with coronavirus disease 2019 (COVID-19). JAMA Cardiol 2020; 5(7):811-8.

20. Bergamaschi L, D'Angelo EC, Paolisso P, et al. The value of ECG changes in risk stratification of COVID-19 patients. Ann Noninvasive Electrocardiol 2021;26(3):1-10.

21. Stefanini GG, Montorfano M, Trabattoni D, et al. STelevation myocardial infarction in patients with COVID-19: clinical and angiographic outcomes. Circulation 2020;2113-6. https://doi.org/10.1161/ CIRCULATIONAHA. 120.047525.

22. Meyer P, Degrauwe S, Van Delden C, et al. Typical takotsubo syndrome triggered by SARS-CoV-2 infection. Eur Heart J 2020;41(19):1860.

23. Jabri A, Kalra A, Reed GW. Incidence of stress cardiomyopathy during the coronavirus disease 2019 pandemic main outcomes and measures. JAMA Netw Open 2020;3(7):e2014780.

24. Okura $\mathrm{H}$. Update of takotsubo syndrome in the era of COVID-19. J Cardiol 2021;77:361-9.

25. Tavazzi G, Pellegrini C, Maurelli M, et al. Myocardial localization of coronavirus in COVID-19 cardiogenic shock. Eur J Heart Fail 2020;22(5):911-5.

26. Sawalha K, Abozenah M, Kadado AJ, et al. Systematic review of COVID-19 related myocarditis: insights on management and outcome. Cardiovasc Revasc Med 2021;23(xxxx):107-13.

27. Ceriani E, Marceca A, Lanfranchi A, et al. Early echocardiographic findings in patients hospitalized for COVID-19 pneumonia: a prospective, single center study. Intern Emerg Med 2021;(0123456789). https://doi.org/10.1007/s11739-021-02733-9.

28. Busana M, Schiavone M, Lanfranchi A, et al. Noninvasive hemodynamic profile of early COVID-19 infection. Physiol Rep 2020;8(20). https://doi.org/ 10.14814/phy2.14628.

29. Stöbe S, Richter S, Seige M, et al. Echocardiographic characteristics of patients with SARS - CoV - 2 infection. Clin Res Cardiol 2020;109(12):1549-66. 
30. Shmueli H, Shah M, Ebinger JE, et al. Left ventricular global longitudinal strain in identifying subclinical myocardial dysfunction among patients hospitalized with COVID-19. IJC Hear Vasc 2021;32:100719.

31. Rothschild E, Baruch G, Szekely Y, et al. The predictive role of left and right ventricular speckle-tracking echocardiography in COVID-19. JACC Cardiovasc Imaging 2020;13(11):2471-4.

32. Faruk O, Hasan B, Barman A, et al. Evaluation of biventricular function in patients with COVID - 19 using speckle tracking echocardiography. Int J Cardiovasc Imaging 2020;(0123456789). https://doi.org/ 10.1007/s10554-020-01968-5.

33. Janus SE, Hajjari J, Karnib M, et al. Prognostic value of left ventricular global longitudinal strain in COVID19. Am J Cardiol 2020;131:134-6.

34. Wibowo A, Pranata R, Astuti A, et al. Left and right ventricular longitudinal strains are associated with poor outcome in COVID-19: a systematic review and meta- analysis. J Intensive Care 2021;9:9.

35. Berlin DA, Gulick RM, Martinez FJ. Severe covid-19. N Engl J Med 2020;383(25):2451-60.

36. Park JF, Banerjee S, Umar S. In the eye of the storm: the right ventricle in COVID-19. Pulm Circ 2020; 10(3).

37. Nishiga M, Wang DW, Han Y, et al. COVID-19 and cardiovascular disease: from basic mechanisms to clinical perspectives. Nat Rev Cardiol 2020;17(9): 543-58.

38. Danzi GB, Loffi M, Galeazzi G, et al. Acute pulmonary embolism and COVID-19 pneumonia: a random association? Eur Heart J 2020;41(19). https://doi.org/10.1093/eurheartj/ehaa254.

39. Paolisso P, Bergamaschi L, D'Angelo EC, et al. Preliminary experience with low molecular Weight Heparin Strategy in COVID-19 patients. Front Pharmacol 2020;11:1-6.

40. Dweck MR, Bularga A, Hahn RT, et al. Global evaluation of echocardiography in patients with COVID19. Eur Heart J Cardiovasc Imaging 2020;21(9): 949-58.

41. Mahmoud-Elsayed HM, Moody WE, Bradlow WM, et al. Echocardiographic findings in patients with COVID-19 pneumonia. Can J Cardiol 2020;36(8): 1203-7.

42. Jain SS, Liu Q, Raikhelkar J, et al. Indications for and findings on transthoracic echocardiography in COVID-19. J Am Soc Echocardiogr 2020;33(10): 1278-84.

43. Kim J, Volodarskiy A, Sultana R, et al. Prognostic utility of right ventricular remodeling over conventional risk stratification in patients with COVID-19. J Am Coll Cardiol 2020;(17):1965-77.

44. Szekely Y, Lichter Y, Taieb P, et al. Spectrum of cardiac manifestations in COVID-19: a systematic echocardiographic study. Circulation 2020;142(4): 342-53.
45. Li Y, Li H, Zhu S, et al. Prognostic value of right ventricular longitudinal strain in patients with COVID-19. JACC Cardiovasc Imaging 2020;13(11):2287-99.

46. Messina A, Sanfilippo F, Milani A, et al. COVID-19related echocardiographic patterns of cardiovascular dysfunction in critically ill patients: a systematic review of the current literature. J Crit Care 2021;65: 26-35.

47. Khan A, James S, Yuan M, Woolery L, Huppertz N. Observations on echocardiographic ndings in patients with COVID-19. Preprint from Research Square. https://doi.org/10.21203/rs.3.rs-58076/v1.

48. Carrizales-Sepúlveda EF, Vera-Pineda R, FloresRamírez R, et al. Echocardiographic manifestations in COVID-19: a review. Hear Lung Circ 2021. https://doi.org/10.1016/j.hlc.2021.02.004.

49. Chong PY, Chui P, Ling AE, et al. Analysis of deaths during the severe acute respiratory syndrome (SARS) epidemic in Singapore: challenges in Determining a SARS diagnosis. Arch Pathol Lab Med 2004;128(2): 195-204.

50. Skulstad H, Cosyns B, Popescu BA, et al. COVID-19 pandemic and cardiac imaging: EACVI recommendations on precautions, indications, prioritization, and protection for patients and healthcare personnel. Eur Heart J Cardiovasc Imaging 2020; 21(6):592-8.

51. Kirkpatrick JN, Mitchell C, Taub C, et al. ASE Statement on protection of patients and echocardiography Service providers during the 2019 Novel coronavirus outbreak: endorsed by the American college of cardiology. J Am Soc Echocardiogr 2020;33(6):648-53.

52. Habib G, Lancellotti P, Antunes MJ, et al. 2015 ESC guidelines for the management of infective endocarditis. Eur Heart J 2015;36(44):3036-7.

53. Baumgartner H, Falk V, Bax JJ, et al. 2017 ESC/ EACTS guidelines for the management of valvular heart disease. Eur Heart J 2017;38(36):2739-91.

54. Furqan MM, Verma BR, Cremer PC, et al. Pericardial diseases in COVID19: a contemporary review. Curr Cardiol Rep 2021;23(7). https://doi.org/10.1007/ s11886-021-01519-x.

55. Kumar R, Kumar J, Daly C, et al. Acute pericarditis as a primary presentation of COVID-19. BMJ Case Rep 2020;13(8):1-3.

56. Dimopoulou D, Spyridis N, Dasoula F, et al. Pericarditis as the main clinical manifestation of covid-19 in adolescents. Pediatr Infect Dis J 2021;40(5):E197-9.

57. Manjili RH, Zarei M, Habibi M, et al. COVID-19 as an acute inflammatory disease. J Immunol 2020;205(1): 12-9.

58. Adler Y, Charron P, Imazio M, et al. 2015 ESC Guidelines for the diagnosis and management of pericardial diseases. Eur Heart J 2015;36(42):2921-64.

59. Imazio M, Brucato A, Lazaros $G$, et al. Anti-inflammatory therapies for pericardial diseases in the 
COVID-19 pandemic: safety and potentiality. J Cardiovasc Med (Hagerstown) 2020;21(9):625-9.

60. Christian M, Lassen H, Skaarup KG, et al. Echocardiographic abnormalities and predictors of mortality in hospitalized COVID - 19 patients : the ECHOVID 19 study. ESC Heart Fail 2020;7(6):4189-97.

61. Ward RP, Lee L, Ward TJ, et al. Utilization and appropriateness of transthoracic echocardiography in response to the COVID-19 pandemic. J Am Soc Echocardiogr 2020;33(6):690-1.

62. McMahon SR, De Francis G, Schwartz S, et al. Tablet-based limited echocardiography to reduce Sonographer scan and decontamination time during the COVID-19 pandemic. J Am Soc Echocardiogr 2020;33(7):895-9.

63. García-Cruz E, Manzur-Sandoval D, Gopar-Nieto R, et al. Transthoracic echocardiography during prone position ventilation: Lessons from the COVID-19 pandemic. J Am Coll Emerg Physicians Open 2020; 1(5):730-6.

64. Elikowski W, Malek-Elikowska M, Fertala N, et al. Tablet-based limited echocardiography at COVID19-dedicated hospital during the pandemic in the context of takotsubo syndrome. Pol Merkur Lekarski 2021;49(289):57-9.
65. Wu S, Wu D, Ye R, et al. Pilot study of robot-assisted teleultrasound based on 5G Network: a new feasible Strategy for early imaging Assessment during COVID-19 pandemic. IEEE Trans Ultrason Ferroelectr Freq Control 2020;67(11):2241-8.

66. Kagiyama N, Shrestha S, Farjo PD, et al. Artificial intelligence: practical primer for clinical Research in cardiovascular disease. J Am Heart Assoc 2019; 8(17):e012788.

67. Bitetto A, Bianchi E, Dondi P, et al. Deep learning detection of cardiac Akinesis in echocardiograms, vol. 12661 LNCS. Springer International Publishing; 2021. p. 503-14. Available at: https://search.bvsalud.org/ global-literature-on-novel-coronavirus-2019-ncov/ resource/es/covidwho-1198421.

68. Cheema BS, Walter J, Narang A, et al. Artificial intelligence-enabled POCUS in the COVID-19 ICU: a new Spin on cardiac ultrasound. JACC Case Rep 2021;3(2):258-63.

69. Zimmerman A, Kalra D. Usefulness of machine learning in COVID-19 for the detection and prognosis of cardiovascular complications. Rev Cardiovasc Med 2020;21(3):345-52. 